\title{
Capacity of Multilayer Diffusion-based Molecular Communication (DBMC) Channel
}

\author{
Saizalmursidi Md Mustam ${ }^{*}$ \\ Department of Communication Engineering, \\ Faculty of Electrical and Electronic Engineering, \\ Universiti Tun Hussein Onn Malaysia (UTHM) \\ 86400 Parit Raja, Batu Pahat \\ Johor, Malaysia \\ saizal@uthm.edu.my \\ UTM-MIMOS Centre of Excellence \\ (CoE) in Telecommunication Technology, \\ Faculty of Electrical Engineering, \\ Universiti Teknologi Malaysia (UTM) \\ 81310 Johor Bahru, Johor, Malaysia

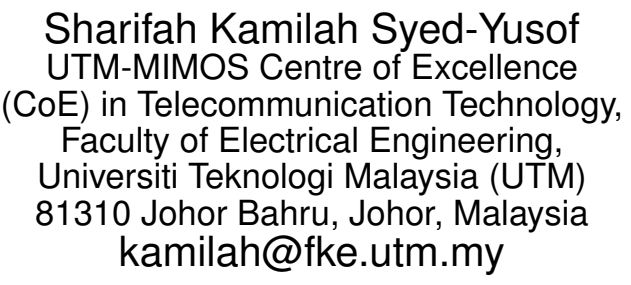 \\ Sharifah Kamilah Syed-Yusof \\ UTM-MIMOS Centre of Excellence \\ oE) in Telecommunication Technology, \\ Universiti Teknologi Malaysia (UTM) \\ kamilah@fke.utm.my \\ Suleiman Zubair \\ UTM-MIMOS Centre of Excellence \\ (CoE) in Telecommunication Technology, \\ Faculty of Electrical Engineering, \\ Universiti Teknologi Malaysia (UTM) \\ 81310 Johor Bahru, Johor, Malaysia \\ zsuleiman2@live.utm.my
}

\begin{abstract}
In diffusion-based molecular communication (DBMC) system, information is encoded in the variation of molecules such as amounts, time shifts, or different molecule types, at a transmitter nanomachine $(\mathrm{TN})$. These molecules are then released and propagated through a channel towards a receiver nanomachine $(\mathrm{RN})$. One of the most important performance matrices in evaluating this system from an information theory perspective is channel capacity. This paper provides a derivation of capacity expression for multilayer DBMC channel in which the propagation of molecules from the TN to the RN through multiple channels follows the Brownian motion and modeled by Fick's equations. The Fourier transforms is employed to convert time to frequency domain functions. The results show that the maximum capacity can be obtained by increasing both the bandwidth and the average transmitted power, and decreasing the TN$\mathrm{RN}$ distance.
\end{abstract}

\section{Categories and Subject Descriptors}

H.1.1 [Information System]: Models and Principles-Systems and Information Theory

\footnotetext{
* Saizalmursidi Md Mustam is currently pursuing a $\mathrm{PhD}$ study in electrical engineering at the Universiti Teknologi Malaysia (UTM), Johor Bahru.
}

\section{General Terms}

Performance, Theory

\section{Keywords}

Nanocommunication, diffusion-based molecular communication (DBMC), multilayer channels model, channel capacity

\section{INTRODUCTION}

In recent times, diffusion-based molecular communication (DBMC) has become a significant research topic among researchers, particularly for biomedical and healthcare applications. In the DBMC, interaction and information sharing among bio-nanomachines in nanonetworks plays an important role in expanding the capability of a single bionanomachine, from performing a simple task to complex tasks. For example, to collect health-related information, synthesis the health condition, and then control the release time as well as the amount of drugs for a treatment.

Recently, researchers have shown considerable interest in the DBMC model for realizing communication among bionanomachines. A comprehensive overview of the DBMC process is addressed in $[1,16]$. The concept of body area nanonetworks with DBMC for healthcare applications is introduced in [4]. Furthermore, the concept of the Internet of Bio-Nano Things (IoBNT), which involving the DBMC model for intra-body communication can be found in [2]. It is expected that from the proposed IoBNT, a healthcare provider can retrieve certain intra-body status parameters such as glucose, sodium, and cholesterol levels, and the presence of unwanted agent through Bio-Nano Things inside the body by using the Internet connection. In addition, existing literatures have shown a great number of efforts in modeling and analyzing the physical layer of DBMC system from the perspective of telecommunication engineering such as; modulation schemes $[9,11,17]$, relaying [7], capacity $[22,23,24]$, detection techniques $[10,11,13]$, throughput [18], noise [21, $22]$, and inter-symbol interference [12, 19]. 
From the information theory perspective, channel capacity is the most important performance metrics which quantifies the maximum amounts of information that can be transferred from the TN to the RN for a given number of transmitted molecules. The work in [24, 23] studied capacity for both the diffusion channel and the ligand-binding reception. In [24], the capacity is developed for continuous transmission of molecules, while in [23] the on-off keying and time slot scheme. Moreover, the general mathematical expression of channel capacity which considered the Fick's diffusion, and the molecular noise can be found in [22]. The capacity is derived for any continuous time signal of molecule transmission as a function of the average thermodynamic power spent by the TN. However, none of the works in literatures investigate the capacity of molecule transmission over multilayer of media. Practically, diffusion can occur through multiple channels inside the human body, such as across an alveolar-blood capillary barrier in the respiratory system, and over the stomach-blood barrier in the absorption process. Therefore, modeling and analyzing the capacity of multilayer DBMC channel is important for supporting future biomedical and the IoBNT applications.

The primary contribution of this paper is the derivation of a closed-form expression of the capacity for a multilayer DBMC channel. In the DBMC system, there are three types of noise, including molecule sampling noise due to unwanted perturbation on the output concentration of the emission process at the TN, molecule counting or diffusion noise due to the randomness in the propagation of molecules through the channel which can impair the estimation of molecule concentration at the $\mathrm{RN}$ [20], and reception noise due to the ligand-receptor binding at the RN [21]. However, any noise is not considered in this work to simplify the analysis and to observe the contributions of Fick's diffusion (channel memory) on the channel capacity.

In this paper, the solution of the mean molecular concentration over an $n$-layer channel derived in [15] and procedures in obtaining the capacity of a DBMC system proposed in [22] are considered to derive a capacity expression for multilayer DBMC channel. In order to make the capacity expression in the general form, the non-origin $\mathrm{TN}$ with continuous or time function transmission of molecules is considered. Then, numerical analyses to evaluate the effect of parameter variation such as an average power transmitted for molecule transmission, bandwidth, distance, and the layer fraction on the capacity of multilayer DBMC channel are presented and discussed. It is expected that the analysis and the modeling of capacity for multilayer DBMC channel can serve as a theoretical basis in understanding the performance of molecule diffusion in multiple channels from an information theory point of view.

The rest of this paper is organized as follows. In Section 2, the considered models of multilayer DBMC channel and assumptions made are presented. The closed-form expressions of mean molecular concentration and capacity for multilayer DBMC channel are also provided in this section. In Section 3 , the numerical results are presented and discussed. Finally, conclusions are given in Section 4 .

\section{THE SYSTEM MODEL}

\subsection{Communication model}

The physical system of multilayer DBMC model consid- ered in this paper is shown in Figure 1. In general, it can be decomposed into three main stages, which are transmission, propagation, and reception. The $\mathrm{TN}$ modulates the transmission of molecules based on time continuous input signal as proposed in [22]. The transmitted molecules are then propagated over multiple channels from the TN to the $\mathrm{RN}$ through a diffusion process via random motion and follows the Fick's second law of diffusion from the regions of high concentration to those of low concentration. The RN reconstructs the message according to the mean concentration of molecules present in a volume sensing area at the $\mathrm{RN}$ location. The modeling of the ligand based reception is beyond the scope of this paper.

The following assumptions are considered in modeling multilayer DBMC channel.

- A point-source type of $\mathrm{TN}$ is located at $\mathbf{r}_{\mathbf{0}}=\left(x_{0}, y_{0}, z_{0}\right)$ and can transmit $Q_{0}$ numbers of the same type of information molecules at time $t=t_{0}$.

- The total concentration of information molecules is considerably lower than the concentration of medium molecules, the interaction between molecules is ignored, and the movement of molecules is independent of each other. Thus, the propagation of molecules in a static medium follows the Brownian motion and can be mathematically modeled using the Ficks laws of diffusion.

- The RN located at $\mathbf{r}=(x, y, z)$ is modeled as a point and a passive observer, and can count the number of molecules within the RN sensing area without any chemical reaction.

- The concentration of molecules within the RN volume sensing area is assumed to be uniform and equal to that counted at the center of the RN $[19,3]$.

- The multilayer DBMC system has no propagation impairments.

- The interlayer between two dissimilar media is considered thin and permeable by the transmitted information molecules. The thinner the interlayer is, the higher is the permeability coefficient of the interlayer. Thus, the transmitted information molecules can easily cross the interlayer. The interlayer also has a unity partition coefficient and has perfect contact between the two layers.

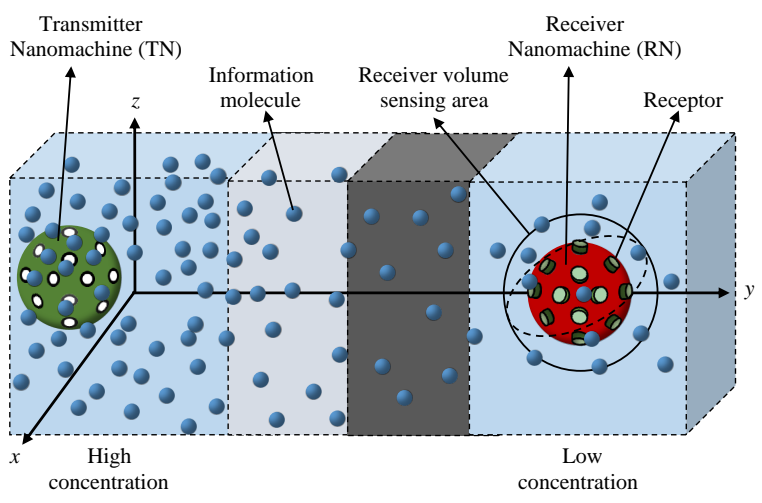

Figure 1: Diagram of multilayer diffusion-based molecular communication (DBMC) physical system. 


\subsection{Propagation of Molecules through Multi- layer Channel by Diffusion}

According to the Fick's second laws of diffusion, the mean molecular concentration in molecules per unit volume at position $\mathbf{r}-\mathbf{r}_{\mathbf{0}}$, changing with time $t$, and propagating across $n$-layer channel is given by [15]:

$$
\frac{\partial C\left(\mathbf{r}-\mathbf{r}_{\mathbf{0}}, t-t_{0}\right)}{\partial t}=\left(\sum_{i=1}^{n} \frac{f_{i}}{D_{i}}\right)^{-1} \nabla^{2} C\left(\mathbf{r}-\mathbf{r}_{\mathbf{0}}, t-t_{0}\right)
$$

where $\frac{\partial}{\partial t}$ is the time derivative operator of the mean concentration of molecules $C\left(\mathbf{r}-\mathbf{r}_{\mathbf{0}}, t-t_{0}\right), \mathbf{r}=(x, y, z)$ and $\mathbf{r}_{\mathbf{0}}=\left(x_{0}, y_{0}, z_{0}\right)$ are the observation and source locations vectors, respectively, $t$ is the time after the initial time $t_{0}$, $i$ represents the number of layers that can take the value of $1,2,3, \ldots n, f_{i}=\frac{r_{i}}{r}$ is the fraction of the $i$-layer $\left(r_{i}\right.$ is the length of the $i$-layer and $r=\left|\mathbf{r}-\mathbf{r}_{\mathbf{0}}\right|$ is the transmission distance), $D_{i}$ is the $i$-layer diffusion coefficient, and $\nabla^{2}$ is the Laplacian operator denotes as $\nabla^{2}=\frac{\partial^{2}}{\partial x^{2}}+\frac{\partial^{2}}{\partial y^{2}}+\frac{\partial^{2}}{\partial z^{2}}$.

The mean concentration of molecules, $C\left(\mathbf{r}-\mathbf{r}_{\mathbf{0}}, t-t_{0}\right)$ in molecules $/ \mathrm{m}^{3}$ can be derived by solving (1) using the Fourier and Laplace transforms for an impulsive transmission of molecules $\left(Q=Q_{0} \delta\left(\mathbf{r}-\mathbf{r}_{0}\right) \delta\left(t-t_{0}\right)\right.$, where both $\delta\left(\mathbf{r}-\mathbf{r}_{\mathbf{0}}\right)$ and $\delta\left(t-t_{0}\right)$ are the Dirac's delta functions of the location and impulsive nature of the source, and $Q_{0}$ is the number of molecules) at time $t_{0}$ in an infinite volume of medium, leading to [15]:

$$
C\left(\mathbf{r}-\mathbf{r}_{\mathbf{0}}, t-t_{0}\right)=\frac{Q_{0} \exp \left(-\frac{\left|\mathbf{r}-\mathbf{r}_{\mathbf{0}}\right|^{2}}{4\left(t-t_{0}\right)} \sum_{i=1}^{n} \frac{f_{i}}{D_{i}}\right)}{\left(4 \pi\left(t-t_{0}\right)\left[\sum_{i=1}^{n} \frac{f_{i}}{D_{i}}\right]^{-1}\right)^{\frac{3}{2}}}
$$

According to the time convolution properties of the delta function and under the assumption that the channel is a linear system, (2) can be written as follows:

$$
C\left(\mathbf{r}-\mathbf{r}_{\mathbf{0}}, t-t_{0}\right)=Q_{0} \delta\left(\mathbf{r}-\mathbf{r}_{\mathbf{0}}\right) \delta\left(t-t_{0}\right) * h\left(\mathbf{r}-\mathbf{r}_{\mathbf{0}} ; t-t_{0}\right)
$$

where $C\left(\mathbf{r}-\mathbf{r}_{\mathbf{0}}, t-t_{0}\right)$ is the output signal, $Q_{0} \delta\left(\mathbf{r}-\mathbf{r}_{\mathbf{0}}\right) \delta(t-$ $\left.t_{0}\right)$ is an arbitrary input signal, the symbol $*$ is a multidimensional convolution operation, and $h\left(\mathbf{r}-\mathbf{r}_{\mathbf{0}} ; t-t_{0}\right)$ is the channel impulse response given by:

$$
h\left(\mathbf{r}-\mathbf{r}_{\mathbf{0}}, t-t_{0}\right)=\frac{\exp \left(-\frac{\left|\mathbf{r}-\mathbf{r}_{\mathbf{0}}\right|^{2}}{4\left(t-t_{0}\right)} \sum_{i=1}^{n} \frac{f_{i}}{D_{i}}\right)}{\left(4 \pi\left(t-t_{0}\right)\left[\sum_{i=1}^{n} \frac{f_{i}}{D_{i}}\right]^{-1}\right)^{\frac{3}{2}}}
$$

Equation (4) is also known as the Green function for transient diffusion equation in 3-dimensional of space.

If the molecule is transmitted by the TN into the medium as a function of time, $Q\left(t-t_{0}\right)$, the solution of (1) gives:

$$
\begin{aligned}
C\left(\mathbf{r}-\mathbf{r}_{\mathbf{0}}, t-t_{0}\right) & =\int_{0}^{\infty} Q(\tau) \frac{e^{-\frac{\left(\mathbf{r}-\mathbf{r}_{0}\right)^{2}}{4 D\left(t-t_{0}-\tau\right)}}}{\left(4 \pi D\left(t-t_{0}-\tau\right)\right)^{\frac{3}{2}}} d \tau \\
& =\delta\left(\mathbf{r}-\mathbf{r}_{\mathbf{0}}\right) Q\left(t-t_{0}\right) * h\left(\mathbf{r}-\mathbf{r}_{\mathbf{0}} ; t-t_{0}\right)
\end{aligned}
$$

where $\tau$ is the variable of integration, $\delta\left(\mathbf{r}-\mathbf{r}_{\mathbf{0}}\right)$ is the Dirac's delta function of the location of the source, $Q\left(t-t_{0}\right)$ is the time function of input signal or the number of molecules transmitted into the medium, $h\left(\mathbf{r}-\mathbf{r}_{\mathbf{0}} ; t-t_{0}\right)$ is the channel impulse response or the Green's function of the Fick's diffusion given in (4) and $C\left(\mathbf{r}-\mathbf{r}_{\mathbf{0}}, t-t_{0}\right)$ is the output signal or the mean molecular concentration.

\subsection{Capacity of Multilayer DBMC Channel}

The maximum transmission rate of communication known as channel capacity is defined as the maximum mutual information between the transmitted and the received signals with respect to the probability density function in all the possible values of the transmitted signal [25]. It can mathematically be represented as follows $[6,25]$ :

$$
C=\max _{f_{x}(x)}\{I(X ; Y)\}
$$

where $C$ is the capacity in a bits per second, $I(X ; Y)$ is the mutual information in bits per second, $X$ is the transmitted or input signal, $Y$ is received or output signal, and $f_{x}(x)$ is the probability density function of all the possible transmitted signal $X$.

\subsubsection{Mutual Information of Multilayer DBMC Chan- nel}

In general, one of the definitions of mutual information $I(X ; Y)$ between the input signal, $X$ and output signal, $Y$ of a communication channel as shown in Figure 2 is given by the following relation $[6]$ :

$$
I(X ; Y)=H(X)-H(X \mid Y)
$$

where $H(X)$ is the entropy per second of the transmitted signal $X, H(X \mid Y)$ is the conditional entropy per second of the transmitted signal $X$ given the received signal $Y$. The graphical representation of Venn diagram is shown in Figure 3 .

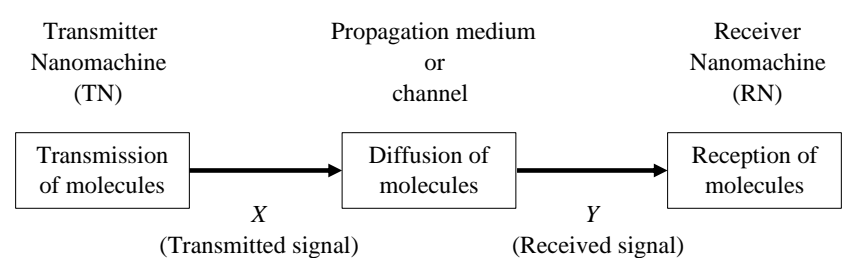

Figure 2: Information-theoretic diagram of multilayer DBMC system.

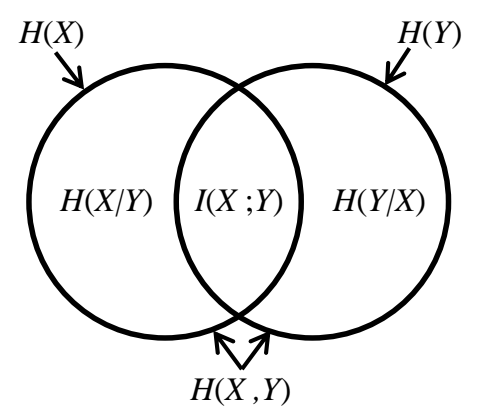

Figure 3: Venn diagrams of the mutual information between the input signal $X$ and output signal $Y[6]$. 
By modeling the time function of the transmitted signal $X$ as a band-limited ensembles function within a bandwidth $W$, the entropy per second $H(X)$ of the transmitted signal $X$ is given by [22]:

$$
H(X)=2 W H(\hat{Q})
$$

where $H(\hat{Q})$ is the entropy of the number of transmitted molecules per time sample of the time function signal $Q(t-$ $t_{0}$ ), and $W$ is the bandwidth of the transmitted signal, $X$. In the traditional communication system, the bandwidth of the transmitted signal is commonly referred to the range of frequencies present in the time function of input signal. In this paper, the bandwidth is defined as the highest frequency of the time function signal, $Q\left(t-t_{0}\right)$.

According to (1), (2) and Figure 2, the multilayer DBMC channel can be classified as a linear and deterministic model. By considering these two properties, the conditional entropy per second of the transmitted signal $X$ given the received signal $Y$ can be obtained from the following relation [22]:

$$
H(X \mid Y)=-\frac{1}{W} \int_{W} \log _{2}\left|h\left(\mathbf{r}-\mathbf{r}_{\mathbf{0}} ; f\right)\right|^{2} d f
$$

where $h\left(\mathbf{r}-\mathbf{r}_{\mathbf{0}} ; f\right)$ is the transfer function Fourier transform of the channel impulse response given in (4).

By performing Fourier transforms, variable substitution, and manipulating the integration part as presented in [14], after that simplification by using de Moivre's formula, (4) can be written in the frequency domain as follows:

$$
h\left(\mathbf{r}-\mathbf{r}_{\mathbf{0}} ; f\right)=\frac{\exp \left(-(1+i)\left|\mathbf{r}-\mathbf{r}_{\mathbf{0}}\right|\left[\pi f \sum_{i=1}^{n} \frac{f_{i}}{D_{i}}\right]^{\frac{1}{2}}\right)}{4 \pi\left|\mathbf{r}-\mathbf{r}_{\mathbf{0}}\right|\left[\sum_{i=1}^{n} \frac{f_{i}}{D_{i}}\right]^{-1}}
$$

Next, by substituting (10) to (9), the conditional entropy per second $H(X \mid Y)$ of the transmitted signal $X$ given the mean molecular concentration $Y$ becomes:

$$
\begin{array}{r}
H(X \mid Y)=2 \log _{2}\left(4 \pi\left|\mathbf{r}-\mathbf{r}_{\mathbf{0}}\right|\left[\sum_{i=1}^{n} \frac{f_{i}}{D_{i}}\right]^{-1}\right)+ \\
\frac{4}{3} \frac{\left|\mathbf{r}-\mathbf{r}_{\mathbf{0}}\right|}{\ln (2)} \sqrt{W \pi \sum_{i=1}^{n} \frac{f_{i}}{D_{i}}}
\end{array}
$$

Then, by applying (8) and (11) to (7), the mutual information of multilayer DBMC channel has the following expression:

$$
\begin{array}{r}
I(X ; Y)=2 W H(\hat{Q})-2 \log _{2}\left(4 \pi\left|\mathbf{r}-\mathbf{r}_{\mathbf{0}}\right|\left[\sum_{i=1}^{n} \frac{f_{i}}{D_{i}}\right]^{-1}\right) \\
-\frac{4}{3} \frac{\left|\mathbf{r}-\mathbf{r}_{\mathbf{0}}\right|}{\ln (2)} \sqrt{W \pi \sum_{i=1}^{n} \frac{f_{i}}{D_{i}}}
\end{array}
$$

\subsubsection{Capacity of Multilayer DBMC Channel}

According to (6), the capacity $C$ of multilayer DBMC channel can be calculated by maximizing the mutual information, $I(X ; Y)$ between the transmitted $X$ and the received $Y$ signals with respect to the probability density function $f_{x}(x)$ of the transmitted signal $X$. The maximum $f_{x}(x)$ is subjected to a constraint on the average thermodynamic power $\bar{P}_{\mathcal{H}}$ or the energy spent by the TN for the transmission of molecules in the medium [22]. Under the consideration of no variations in the pressure and the volume of the channel, the maximum entropy $H(\hat{Q})$ in the number of transmitted molecules per time sample is given by [22]:

$$
H(\hat{Q})=\left(1+\log _{2}\left[\frac{\bar{P}_{\mathcal{H}}}{3 W K_{b} T}\right]\right)
$$

where $K_{b}$ is the Boltzmann constant, and $T$ is the absolute temperature of the physical system.

By substituting (13) to (12), the capacity of multilayer DBMC channel can be expressed as follows:

$$
\begin{aligned}
C=2 W\left(1+\log _{2}\left[\frac{\bar{P}_{\mathcal{H}}}{3 W K_{b} T}\right]\right)- \\
2 \log _{2}\left(4 \pi\left|\mathbf{r}-\mathbf{r}_{\mathbf{0}}\right|\left[\sum_{i=1}^{n} \frac{f_{i}}{D_{i}}\right]^{-1}\right)- \\
\frac{4}{3} \frac{\left|\mathbf{r}-\mathbf{r}_{\mathbf{0}}\right|}{\ln (2)} \sqrt{W \pi \sum_{i=1}^{n} \frac{f_{i}}{D_{i}}}
\end{aligned}
$$

where $W$ is the bandwidth of the transmitted signal, $X, \bar{P}_{\mathcal{H}}$ is the average thermodynamic power, $K_{b}$ is the Boltzmann constant, and $T$ is the absolute temperature of the system, $\left|\mathbf{r}-\mathbf{r}_{\mathbf{0}}\right|$ is the transmission distance, $i$ represents the number of layers that can take the value of $1,2,3, \ldots n, f_{i}=\frac{r_{i}}{r}$ is the fraction of the $i$-layer $\left(r_{i}\right.$ is the length of the $i$-layer and $\left.r=\left|\mathbf{r}-\mathbf{r}_{\mathbf{0}}\right|\right)$, and $D_{i}$ is the $i$-layer diffusion coefficient.

\section{RESULTS AND DISCUSSION}

In this section, numerical analysis is performed to evaluate the capacity of multilayer DBMC channel using (14). For simplicity, the air-water-blood plasma channel is chosen as the multilayer DBMC channel. The simplified cross sectional channel as shown in Figure 4 is chosen based on the alveolar-blood barrier in the respiration system. The temperature is set to be the normal body temperature of $37^{\circ} \mathrm{C}$. The other set of parameters are given in Table 1 . However, the chosen values are only a reference value.

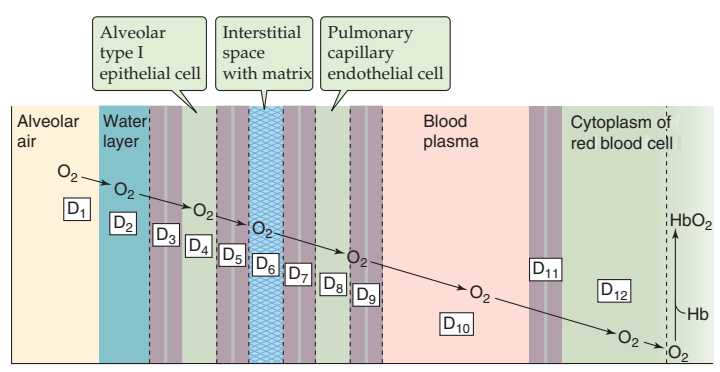

(a)

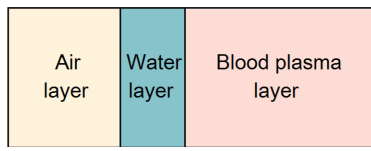

(b)

Figure 4: Multilayer diffusion: (a) alveolar-blood barrier [5], and (b) the simplified multilayer representation. 
Table 1: A Common Set of Parameters.

\begin{tabular}{|l|l|}
\hline Parameter & Value \\
\hline Transmission distance, $r$ & 1 to $500 \mu \mathrm{m}$ \\
\hline Number of layer, $n$ & 3 \\
\hline Fraction of each layer, $f_{i}$ & $f_{1}=f_{2}=f_{3}=1 / 3$ \\
\hline The average TN power, $\bar{P}_{\mathcal{H}}$ & 0.1 to $10 \mathrm{pW}$ \\
\hline Transmission bandwidth, $W$ & 20 to $40 \mathrm{~Hz}$ \\
\hline Radius of molecule $(\backsim$ an Oxygen atom) & $6.6 \times 10^{-11} \mathrm{~m}[8]$ \\
\hline Boltzmann constant, $K_{b}$ & $1.3806504214 \times 10^{-23} \mathrm{~J} / \mathrm{K}$ \\
\hline Viscosity of air, $\eta_{\text {air }}$ at $37^{\circ} \mathrm{C}$ & $0.01894 \times 10^{-3} \mathrm{~kg} / \mathrm{ms}[8]$ \\
\hline Viscosity of water, $\eta_{\text {water }}$ at $37^{\circ} \mathrm{C}$ & $0.6915 \times 10^{-3} \mathrm{~kg} / \mathrm{ms}[8]$ \\
\hline Viscosity of blood plasma, $\eta_{\text {plasma }}$ at $37^{\circ} \mathrm{C}$ & $1.1 \times 10^{-3} \mathrm{~kg} / \mathrm{ms}[8]$ \\
\hline Diffusion coefficient of air, $D_{\text {air }}$ & $1.82 \times 10^{-7} \mathrm{~m}^{2} / \mathrm{s}$ \\
\hline Diffusion coefficient of water, $D_{\text {water }}$ & $4.98 \times 10^{-9} \mathrm{~m}^{2} / \mathrm{s}$ \\
\hline Diffusion coefficient of blood plasma, $D_{\text {plasma }}$ & $3.13 \times 10^{-9} \mathrm{~m}^{2} / \mathrm{s}$ \\
\hline
\end{tabular}

\subsection{Capacity Versus Bandwidth for Different Values of Transmission Distance}

Figure 5 shows the capacity of multilayer DBMC channel against the transmission bandwidth for the air-water-blood plasma with different values of transmission distance, $r$ (50 $\mu \mathrm{m}$ of step size). The average power of $\mathrm{TN}$ is fixed at $0.1 \mathrm{p}$ W. Each layer is considered to have an equal fraction. It can be seen that, increasing the transmission bandwidth from 20 to $40 \mathrm{~Hz}$ increases the capacity of multilayer DBMC channel from 0.85 to 1.55 kilobits per second, at the transmission distance of $50 \mu \mathrm{m}$. The same trend of constant capacity increment by a factor of around 1.8 to 1.9 can also be observed at the other transmission distance. The upper line (the line with a circle marker) refers to the transmission distance of $50 \mu \mathrm{m}$, whereas the lower line (the dashed-line with rectangular markers) represents the capacity at $500 \mu \mathrm{m}$ of TN-RN distance. Doubling the bandwidth, increases the first and the third terms of (14) by factor 1.9 and $\sqrt{2}$, respectively. It can be concluded that the wider the transmission bandwidth, the higher the channel capacity.

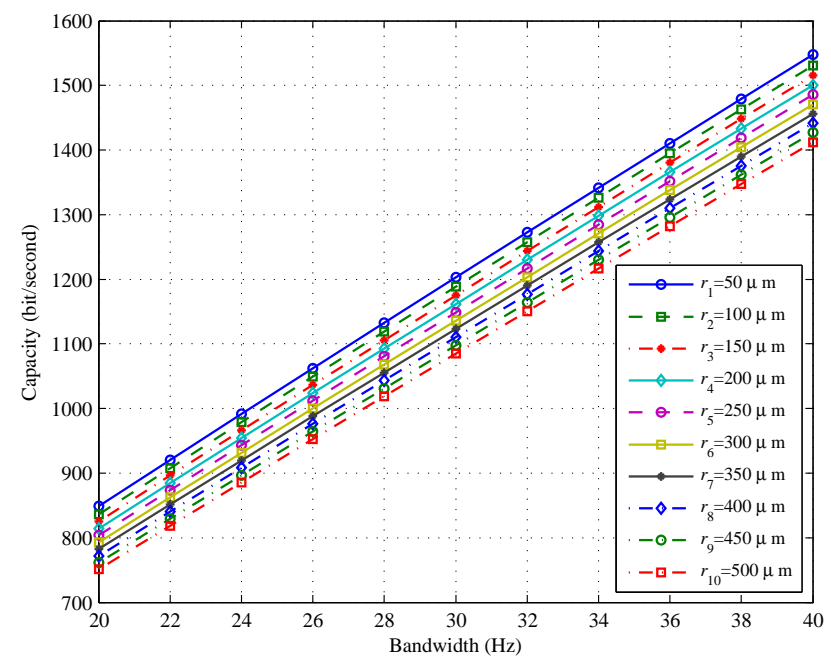

Figure 5: Capacity for various TN-RN distances in air-water-blood plasma as a function of bandwidth.

\subsection{Capacity Versus Transmission Distance for Different Values of Bandwidth}

In addition, increasing the transmission distance decreases the capacity of multilayer DBMC channel. This is demonstrated in Figure 6. In this numerical evaluation, the average transmitted power is set to be $0.1 \mathrm{pW}$. According to Figure 6 , for the transmission bandwidth of $38 \mathrm{~Hz}$ (the dashedline with rectangular markers), the capacity value decreases from 1.48 to 1.32 kilobits per second as the TN-RN distance increases from $50 \mu \mathrm{m}$ to $500 \mu \mathrm{m}$. The other curves of Figure 6 refers to the variation of bandwidth from 20 to 38 $\mathrm{Hz}$ also show a constant decrement in capacity as the distance increased. As it can be seen in (14), the decrement in capacity value is mainly contributed by the third term of (14) where the capacity value is directly proportional to the transmission distance, $\left|\mathbf{r}-\mathbf{r}_{\mathbf{0}}\right|$. The second term of (14) shows only a small variation. This trend shows that the maximum capacity can be achieved with short transmission distance and high transmission bandwidth.

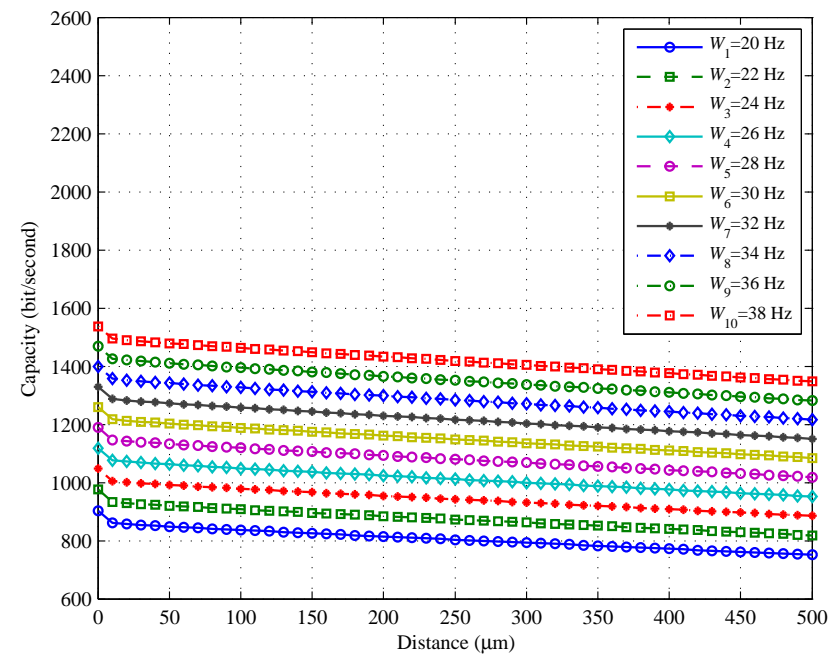

Figure 6: Capacity for various transmission bandwidth as a function of TN-RN distance over airwater-blood plasma channel. 


\subsection{Capacity Versus Transmission Distance for Different Values of Layer Fraction}

The effect of different layer fractions on the capacity of multilayer DBMC channel is shown in Figure 7. The transmission distance and the transmission bandwidth are set to be $50 \mu \mathrm{m}$ and $40 \mathrm{~Hz}$, respectively. By changing the fractions of air-water-blood plasma layer fraction from $\left(f_{1}=\right.$ $1 / 3, f_{2}=1 / 3$, and $\left.f_{3}=1 / 3\right)$ to $\left(f_{1}=4 / 6, f_{2}=1 / 6\right.$, and $\left.f_{3}=1 / 6\right)$ increases the capacity value ranging from 1.55 to 1.42 kilobits per second to 1.55 to 1.45 kilobits per second as the distance increases from $50 \mu \mathrm{m}$ to $500 \mu \mathrm{m}$. However, the capacity decreases from the range of 1.55 to 1.39 kilobits per second when the layer fraction changes from $\left(f_{1}=1 / 3, f_{2}=1 / 3\right.$, and $\left.f_{3}=1 / 3\right)$ to $\left(f_{1}=1 / 6, f_{2}=1 / 6\right.$, and $\left.f_{3}=4 / 6\right)$. According to (14), the last two terms will increase with respect to the transmission distance as well as the layer fraction. As the transmission distance and layer fraction increases, these two terms increases accordingly, in consequence, the capacity value has a smaller deviation when the TN-RN distance is shorter but higher for larger transmission distance.

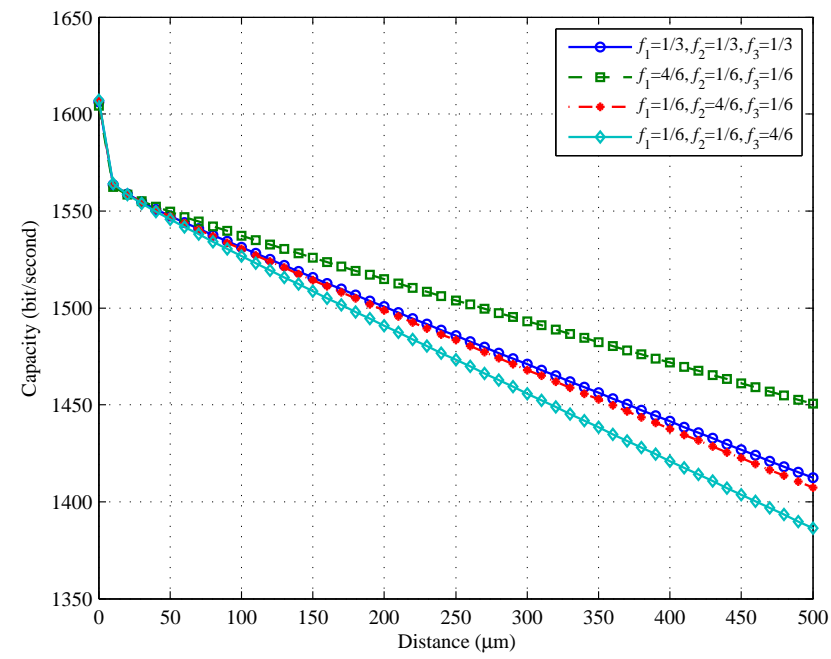

Figure 7: Capacity for various layer fraction as a function of TN-RN distance over air-water-blood plasma channel.

\subsection{Capacity Versus Transmission Bandwidth for Different Values of the Average Trans- mitted Power}

Figure 8 shows the capacity for different values of the average transmitted power ( 1 to $10 \mathrm{pW}$ ) spent by the $\mathrm{TN}$ for molecule transmission, as a function of bandwidth from 20 to $40 \mathrm{~Hz}$. The transmission distance is set to $50 \mu \mathrm{m}$. It can be observed that, the higher the value of average transmitted power, the higher the channel capacity. For the bandwidth ranging from 20 to $40 \mathrm{~Hz}$, the capacity increases from 0.98 to 1.81 kilobits per second with $1 \mathrm{pW}$ of the average transmitted power, and from 1.11 to 2.08 kilobits per second for $10 \mathrm{pW}$ of the average transmitted power. This is because of increment in the averaged transmitted power increases only the first positive term of (14). In consequence, the capacity increases as the averaged transmitted power increase.

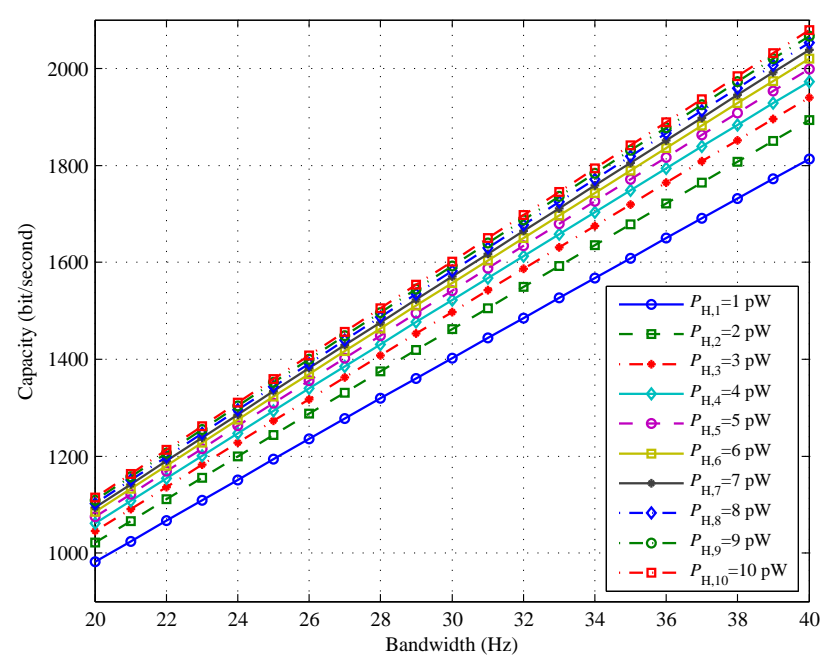

Figure 8: Capacity for various average transmitted power as a function of bandwidth over air-waterblood plasma channel.

\section{CONCLUSIONS}

This paper presented the derivation of a closed-form expression of capacity in a multilayer DBMC channel. The capacity was evaluated for molecule transmission through airwater-blood plasma channel representing a multilayer diffusion in the respiratory system. The numerical results show that, the wider the transmission bandwidth, the higher the channel capacity, and the shorter transmission distance, the higher the channel capacity. Increasing the average transmitted power increases the channel capacity as well. These results show an interesting insight as a theoretical basis in understanding the performance of molecule diffusion in multiple channels from an information theory point of view. Future work will include studying a multilayer DBMC channel by taking into account both the transmission impairments and the ligand-binding reception at the $\mathrm{RN}$.

\section{ACKNOWLEDGMENTS}

The first author would like to thank the Ministry of Education, Malaysia, and the University Tun Hussein Onn Malaysia (UTHM) for the financial support in the form of a doctoral scholarship and monthly allowances. The author would also like to thank the UTM-MIMOS Centre of Excellence in Communication Technology for providing a very conducive workspace during the period of 2013 to 2016 to carry out this research work. This research work is financially supported by the UTM GUP Research Grant (Vot No: Q.J1300000.2509.07H04).

\section{REFERENCES}

[1] I. F. Akyildiz, F. Brunetti, and C. Blaquez. Nanonetworks: A new communication paradigm. Computer Networks, 52(12):2260-2279, August 2008.

[2] M. B. S. Akyildiz, Ian F. Pierobon and Y. Koucheryavy. The internet of bio-nano things. IEEE Communications Magazine, 53(3):32-40, March 2015 . 
[3] B. Atakan and O. B. Akan. Deterministic capacity of information flow in molecular nanonetworks. Nano Communication Networks, 1(1):31-42, 2010.

[4] B. Atakan, O. B. Akan, and S. Balasubramaniam. Body area nanonetworks with molecular communications in nanomedicine. IEEE Communications Magazine, 50(1):28-34, January 2012.

[5] W. F. Boron and E. L. Boulpaep. Medical Physiology. Saunders, Elsevier, Philadelphia, PA, 2nd edition, 2011.

[6] T. M. Cover and J. A. Thomas. Elements of Information Theory. John Wiley and Sons, New York, 2nd edition, 2006.

[7] A. Einolghozati, M. Sardari, and F. Fekri. Relaying in diffusion-based molecular communication. In 2013 IEEE International Symposium on Information Theory Proceedings (ISIT), pages 1844-1848, Istanbul, Turkey, July 2013. IEEE.

[8] J. R. A. Freitas. Nanomedicine, Volume I: Basic capabilities. Landes Biosciences, Georgetown, Texas, U.S.A, 1999.

[9] M. S. Kuran, H. B. Yilmaz, T. Tugcu, and I. F. Akyildiz. Modulation techniques for communication via diffusion in nanonetworks. In 2011 IEEE International Conference on Communications (ICC), pages 1-5, Kyoto, Japan, June 2011. IEEE.

[10] I. Llatser, A. Cabellos-Aparicio, M. Pierobon, and E. Alarcon. Detection techniques for diffusion-based molecular communication. IEEE Journal on Selected Areas in Communications, 31(12):726-734, December 2013.

[11] M. U. Mahfuz, D. Makrakis, and H. T. Mouftah. On the characterization of binary concentration-encoded molecular communication in nanonetworks. Nano Communication Networks, 1(4):289-300, December 2010.

[12] M. U. Mahfuz, D. Makrakis, and H. T. Mouftah. Characterization of intersymbol interference in concentration-encoded unicast molecular communication. In 2011 24th Canadian Conference on Electrical and Computer Engineering (CCECE), pages 164-168, Niagara Falls, Canada, 2011. IEEE.

[13] M. U. Mahfuz, D. Makrakis, and H. T. Mouftah. Sampling based optimum signal detection in concentration-encoded molecular communication: receiver architecture and performance. In 6 th International Conference on Bio-inspired Systems and Signal Processing (BIOSIGNALS-2013), pages 372-376, Barcelona, Spain, February 2013. INSTICC.
[14] A. Mandelis. Diffusion-Wave Fields: Mathematical Methods and Green Functions. Springer Science+Business Media, New York, 2001.

[15] S. Md Mustam, S. K. Syed Yusof, and S. Nejatian. Multilayer diffusion-based molecular communication. Transactions on Emerging Telecommunications Technologies, DOI:10.1002/ett.2935, 2015.

[16] T. Nakano, M. J. Moore, W. Fang, A. V. Vasilakos, and S. Jianwei. Molecular communication and networking: Opportunities and challenges. IEEE Transactions on Nano Bioscience, 11(2):135-148, June 2012.

[17] T. Nakano, Y. Okaie, and L. Jian-Qin. Channel model and capacity analysis of molecular communication with brownian motion. IEEE Communications Letters, 16(6):797-800, June 2012.

[18] T. Nakano, Y. Okaie, and A. V. Vasilakos. Throughput and efficiency of molecular communication between nanomachines. In 2012 IEEE Wireless Communications and Networking Conference (WCNC), pages 704-708, Shanghai, April 2012. IEEE.

[19] A. Noel, K. C. Cheung, and R. Schober. Improving receiver performance of diffusive molecular communication with enzymes. IEEE Transactions on NanoBioscience, 13(1):31-43, March 2014.

[20] M. Pierobon and I. F. Akyildiz. Diffusion-based noise analysis for molecular communication in nanonetworks. IEEE Transactions on Signal Processing, 59(6):2532-2547, 2011.

[21] M. Pierobon and I. F. Akyildiz. Noise analysis in ligand-binding reception for molecular communication in nanonetworks. IEEE Transactions on Signal Processing, 59(9):4168-4182, September 2011.

[22] M. Pierobon and I. F. Akyildiz. Capacity of a diffusion-based molecular communication system with channel memory and molecular noise. IEEE Transactions on Information Theory, 59(2):942-954, February 2013.

[23] L. Qiang and Y. Kun. Channel capacity analysis of a diffusion-based molecular communication system with ligand receptors. International Journal of Communication Systems, 28(8):1508-1520, May 2015.

[24] L. Qiang, Y. Kun, and H. Peng. Channel capacity analysis for molecular communication with continuous molecule emission. In 2013 International Conference on Wireless Communications $\mathcal{E}$ Signal Processing (WCSP), pages 1-6. IEEE, October 2013.

[25] C. E. Shanon. A mathematical theory of communication. Bell System Technical Journal, 27:379-423, 1948. 